\title{
Correspondence
}

Editor: Ian Pullen

Contents: Personality disorder and self-report questionnaire/Combination treatment of depression/ Incidence and epidemiology of schizophrenia in Denmark/Origins of delusions/The 'myth' of suicide prevention/Professional and lay opinions on multiple personality disorder/Cannabis and the PSE/ Conversion mnemonic/Gender difference of schizophrenia in seasonal admissions in Scotland/ Estimating premorbid IQ in schizophrenia/Catatonia and not neuroleptic malignant syndrome/'Mabi bark tea' consumption and psychosis?/Simultaneous kidney disease and manic-depressive psychosis/ Down's syndrome, longevity, and Alzheimer's disease/Eating disorders in Hong Kong.

\section{Personality disorder and self-report questionnaire}

SIR: Dowson (Journal, September 1992, 161, 344-352) has provided further important evidence that the assessment of DSM-III-R personality disorder by self-report instruments such as the Personality Diagnostic Questionnaire (PDQ; or a modified version of the instrument) is invalid and that such instruments should not be employed in future studies. Unfortunately, he did not make this interpretation from his findings and encourages further use of the PDQ in clinical settings. His encouragement to use the screening test for comorbid personality disorders when patients are not able or willing to complete a questionnaire was puzzling after so clearly demonstrating that self-report and informant scores show so little resemblance.

His reference to the previous worrying findings of overdiagnosis by the PDQ was not as clear as it should have been. For the record, Hyler et al (1989) administered the instrument to 552 patients and compared the findings with clinicians' diagnoses. Those found by PDQ to have a personality disorder showed a mean of 3.0 Axis-II categories per patient compared with only 1.2 when diagnosed by a clinician. More importantly, the PDQ continued to diagnose a mean of 1.7 categories in subjects where clinicians diagnosed none.

Dowson is not correct in assuming that current interviewing instruments for Axis-II disorders are too long for routine clinical use. In-patients admitted to the East London Forensic Psychiatry Services are regularly diagnosed with the SCID-II administered by junior medical staff who are trained in its use at the beginning of each rotational placement in forensic psychiatry. Encouragement is given to supplement information obtained at interview with observations by patients' relatives and nursing staff. It is essential to improve training in the diagnosis of personality disorders in a service where $25 \%$ of patients have one or more of these conditions as their primary diagnosis. Clinical experience in forensic psychiatry indicates that there should be no short cuts to obtaining accurate personality disorder diagnoses. Both treatment and long-term management of mentally abnormal offenders can have adverse consequences when wrongly influenced by overdiagnosis or the diagnosis of a non-existent condition based on poor quality diagnostic techniques.

No study of schizophrenia would be accepted for publication where the researchers had handed out a modified PSE or SADS-L to be completed by the subjects. Similarly, no such instrument would be adopted by other researchers if clinicians could find the condition in only half the cases so diagnosed. Caron \& Rutter (1991) have argued that future study of comorbidity is of major importance. The use of instruments that grossly overdiagnose Axis-II psychopathology will lead to few developments in this area.

Caron, C. \& RUTter, M. (1991) Co-morbidity in child psychopathology: concepts, issues and research strategies. Journal of Child Psychology and Psychiatry, 32, 1063-1080.

Hyler, S. E., Rieder, R. O., Williams, J. B. W., et al (1989) A comparison of clinical and self-report diagnoses of DSM-III personality disorders in 552 patients. Comprehensive Psychiatry, 30, 170-178.

JEREMY COID

Department of Psychological Medicine

St Bartholomew's Hospital

London ECI

AUTHOR's REPLY: The different versions of the PDQ self-report questionnaire for DSM-III-R personality disorder (PD) have face validity in respect of DSM-III-R and have shown various other types of 